\title{
Silicon Neuronal Network with Excitability Neurons for Edge Detection
}

\author{
J. $\mathrm{Li}^{1, \mathrm{a}^{*}}$ and B. Liu ${ }^{2, \mathrm{~b}}$ \\ ${ }^{1}$ College of Electronic Engineering, Xi'an Shiyou University, Xi'an, Shannxi Province, China \\ ${ }^{2}$ Key Laboratory of Intelligent Perception and Image Understanding of Ministry of Education, \\ International Research Center for Intelligent Perception and Computation, Xidian University, Xi'an, \\ Shannxi Province, 710071, China
}

alijing@xsyu.edu.cn, bliub@xidian.edu.cn

\begin{abstract}
Keywords: silicon neuronal network; digital spiking silicon neuron; silicon synapse; receptive field; edge detection
\end{abstract}

\begin{abstract}
Inspired by the human vision system, neuromorphic vision systems simulating the mechanism of signal processing in retina are extensively investigated. This paper describes a silicon neuronal network with bio-inspired structure detects the edge information in image. The digital spiking silicon neuron (DSSN) and silicon synapse models are used to reproduce the neuron, synapse and their dynamic behaviors. The biological receptive fields are realized by the combination of excitatory and inhibitory synapses. Our silicon neuronal network is optimized for the hardware implementation. It is expected to have low hardware consumption and high running speed. The simulation results show that edge detection is successful in our network.
\end{abstract}

\section{Introduction}

Neuromorphic system performs based on the bio-inspired mechanism with efficient computation, high running speed and low power consumption. Biological studies indicate that the nerve cells in retina generate light sensitive spike trains response to intensity, temporal and spatial context [1].

Neuromorphic vision systems implementing the vision algorithms of retina and cortex on electronic circuits are extensively studied. A silicon retina applied as the intraocular retina prostheses mimics the role of neurons in retina and generates spiking signals [2]. Reference [3] introduced a dynamic vision sensor (DVS) on CMOS circuits which runs in the real-time and is sensitive to the temporal changes of the scene. The asynchronous spikes generated by the DVS are processed by the retina-inspired algorithms on software and field-programmable gate array (FPGA) infrastructure [4,5]. Reference [6] designed another CMOS-based vision sensor extracting the temporal contrast and grey level information. Combined with this vision sensor, [7] investigated an algorithm of image segmentation on FPGA device. Reference [8] proposed a fully digital solution of neuromorphic vision which runs in parallel structure with the low power consumption and the speedup computation. However, the neuron behaviors do not be considered in the above neuromorphic vision systems. The integrate-and-fire (IF) model in [9] describing the phenomenon of a spike with one simple differential equation are considered in many neuromorphic systems for its compact hardware implementation. Reference [10] realized a human vision similar neuromorphic with IF model based spiking neural network on graphic processing units (GPU). In our work, we will introduce a silicon neuronal network with complex neuron model to simulate the behaviors of neurons and neural network in retina. Our silicon neuron network is built on FPGA device and designed for the edge detection.

This paper is organized as follows: the information processing in vision sensor is explained in section II. Section III introduces the architecture of our silicon neuronal network. The neuron and synapse models are detailed. Their implementations are presented. Section IV shows the simulation results for edge detection and comparison with Laplacian operation. Discussion and the future plan are presented in section $\mathrm{V}$. 


\section{Vision Sensor}

The retina is a multi-layered tissue and lines the back of the eye. It converts rays of light into electrical signals which are fed to the cortex by the optic nerve. The neurons in retina are organized into 5 types as shown in Fig.1. The photoreceptors in the first layer convert lights into nerve impulses which are transmitted to the bipolar neurons in the second layer and then to the ganglion neurons in the third layer. The neurons in the intermediate layers serve the space smoothing function because they connect neighbors. The horizontal neurons, for instance, receive large range of stimuli from the photoreceptors and transmit them to the bipolar neurons. And the amacrine neurons connect between the bipolar and the ganglion neurons.

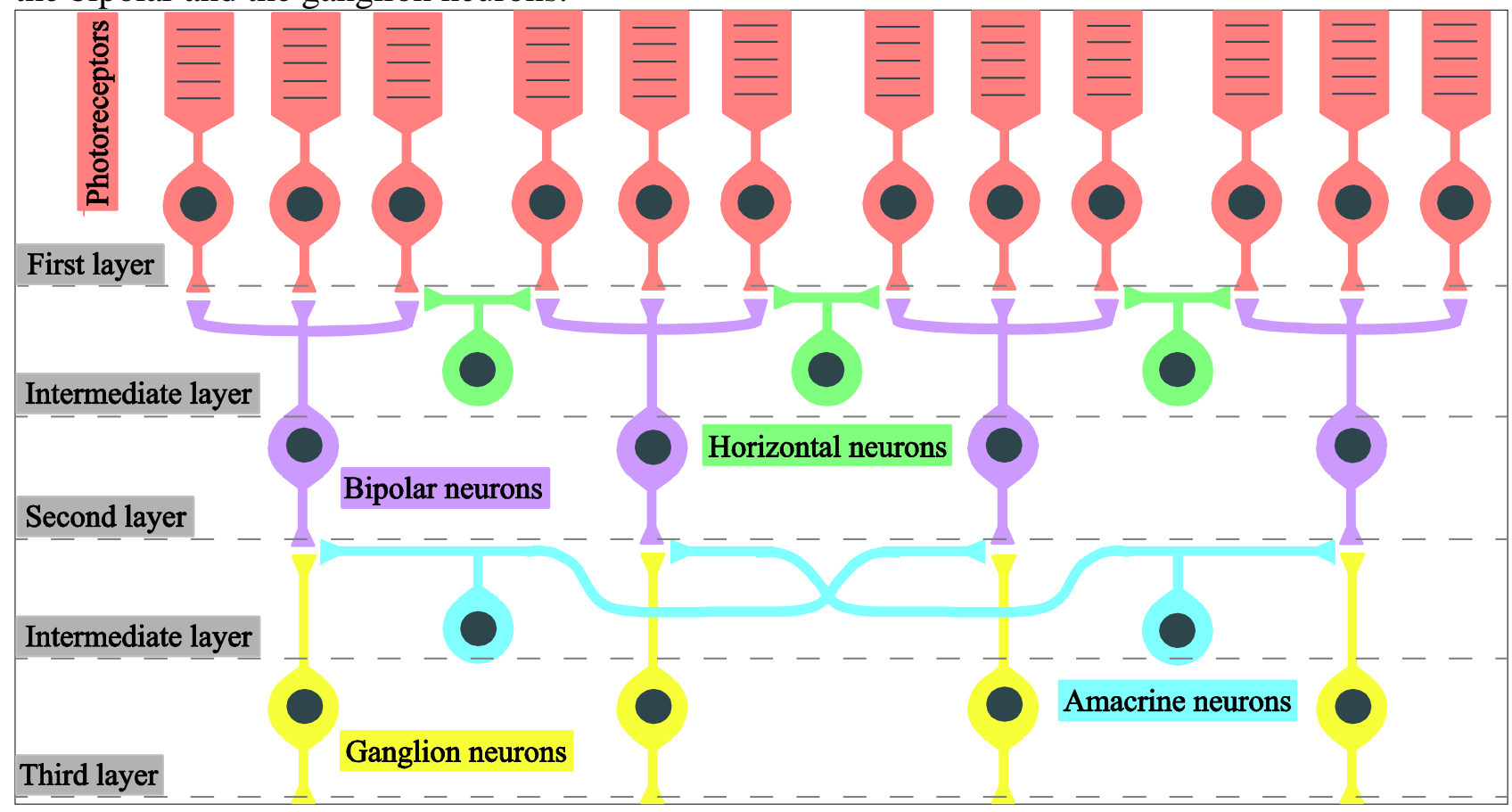

Fig. 1. Section of retina.

The receptive field of a neuron refers to an area in the field of vision in which the stimulus will alter the activity of that neuron. Figure 2 shows the receptive fields and activities of three kinds of neurons: photoreceptor, bipolar neuron and ganglion neuron. The receptive field of photoreceptor corresponds to its location in the retina (A). The activity of photoreceptor shows a logarithm output (D). The receptive field of bipolar neuron is a concentric circular which is caused by two stimulus inputs coming from the photoreceptors and the horizontal neurons (B). This kind of receptive field contains center and surrounding regions which respond to the ray of light in opposite ways. The light in center trends to increase (decrease) the membrane potential which refers to the On-center (Off-center) bipolar neurons. Figure 2(E) shows the activities of these two types of neurons. When the center receives the light stimulus and the surrounding does not, the membrane potential is increased (decreased) in On-center (Off-center) neuron which refers to depolarization (hyperpolarization). And their activities reverse when the light stimulus illuminates in the surrounding not the center. The receptive field of ganglion neuron is similar to that of bipolar neuron(C). However, their activities are different. The ganglion neuron responds to the ray of light by firing frequency not the membrane potential in bipolar neuron. The firing frequency increases (decreases) for On-center (Off-center) neuron if only the center receives stimulus. And their activities reverse like the bipolar neuron. 

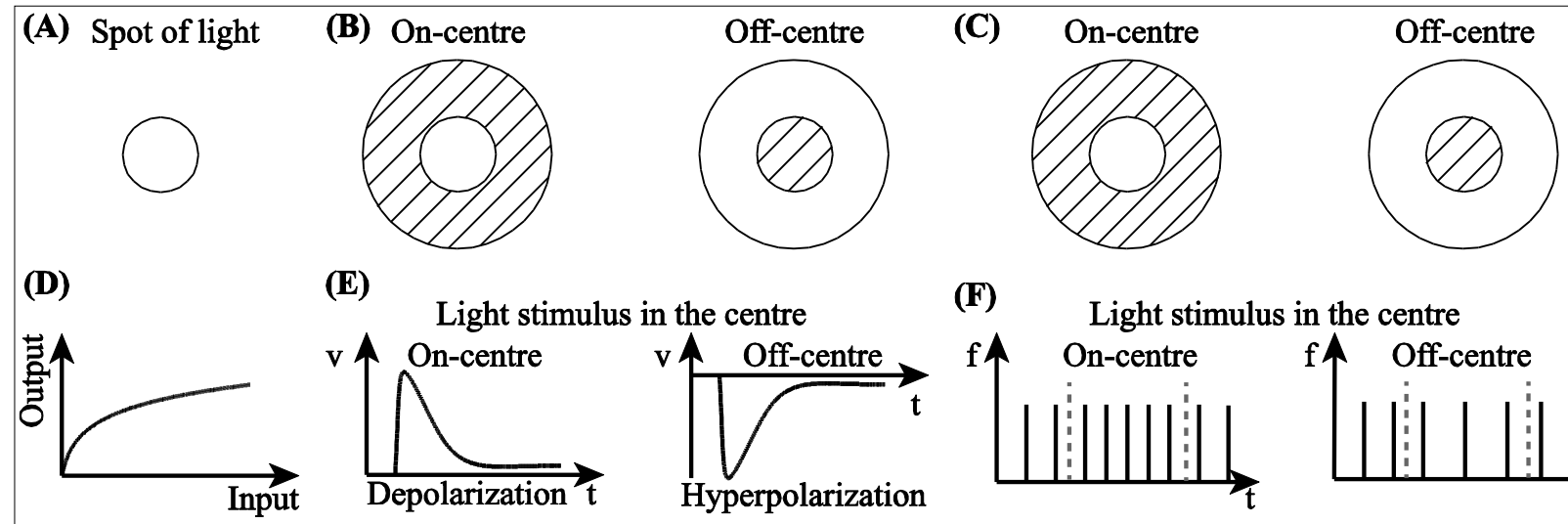

(E)
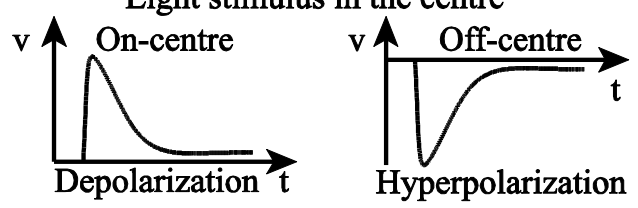

Light stimulus in the surround
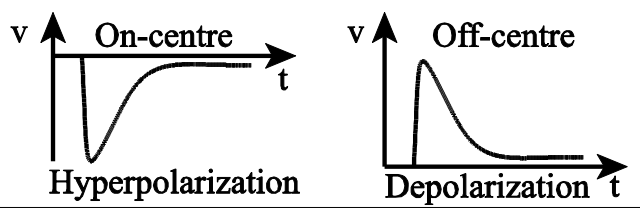

(F)

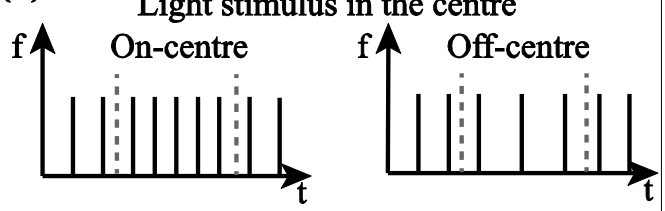

Light stimulus in the surround
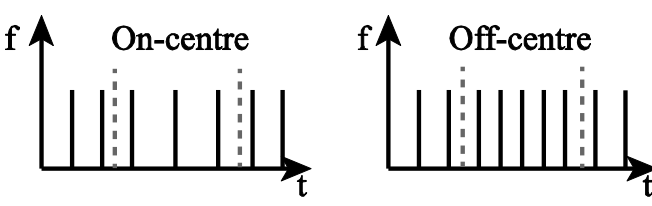

Fig. 2. Receptive fields and activities of photorecptor(A,D), bipolar neuron(B,E) and ganglion neuorn $(\mathrm{C}, \mathrm{F})$. Parameters $\mathrm{v}$ in $(\mathrm{E})$ and $\mathrm{f}$ in $(\mathrm{F})$ are membrane potential and frequency, respectively.

\section{Silicon Neuronal Network}

\section{Architecture}

Our silicon neuronal network reproduces neurons in the retina and their signal processing ways for the efficient image process. Because the receptive fields of bipolar and ganglion neurons are same as concentric circular, the horizontal and amacrine neurons serve the same function of space smooth, we consider the ganglion and amacrine neurons only in our network and suppose they receive stimuli directly from the photoreceptors. Figure 3 shows the structure of our silicon neuronal network. The image in the input layer is presented to the retina. The neurons $\left(\mathrm{N}^{1}\right)$ in the layer 1 receive stimulus input which corresponds to the value of each pixel in the image. And (i,j) is the coordinate of pixel. The neurons $\left(\mathrm{N}^{2}\right.$ on and $\mathrm{N}^{2}$ off $)$ in the layer 2 represent the ganglion neurons with the same size of the image. Their receptive fields correspond to synaptic matrices $\left(\mathrm{W}_{\text {on }}\right.$ and $\left.\mathrm{W}_{\text {off }}\right)$ with excitatory and inhibitory synapses which locate in center and surrounding respectively for the On-center neuron and locate in the opposite way for the Off-center neuron. The edge is detected due to $\mathrm{N}^{2}$ on and $\mathrm{N}^{2}$ off respond to the different stimuli in center and surrounding.

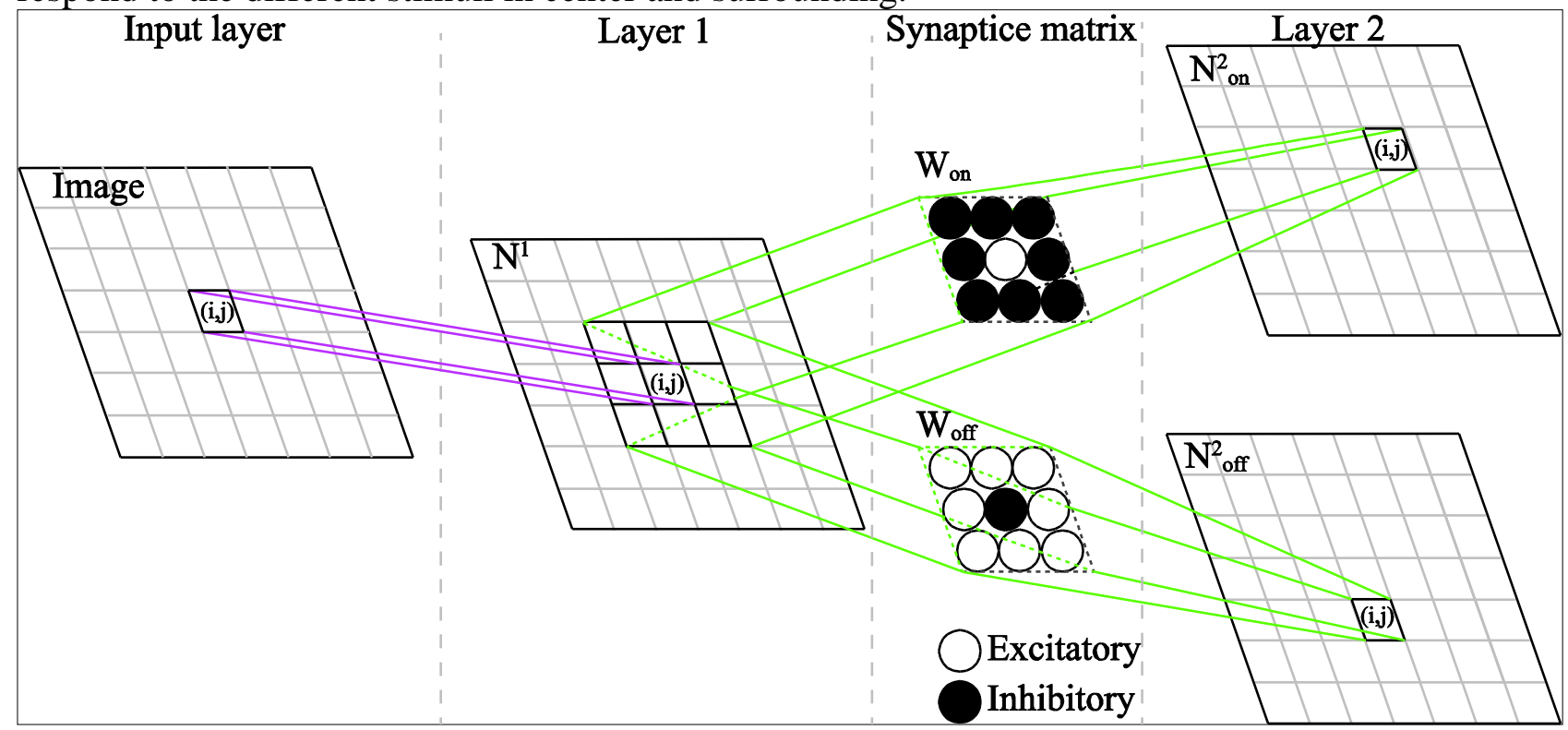

Fig. 3. Structure of silicon neuronal network. 


\section{Silicon Neuron and Synapse Models}

The digital spiking silicon neuron (DSSN) is a 2-variable silicon neuron model which describes various neuron behaviors from the mathematical viewpoint [11]. It can reproduce both the Class I and II neurons in Hodgkin's classification [12]. The Class I neuron starts to response stimulus from a zero firing frequency. While the Class II neuron starts from a non-zero frequency. The DSSN model can show frequency sensitivity like the ganglion neurons in retina. The model is governed by following equations:

$$
\begin{gathered}
\frac{d v_{i, j}^{x}}{d t}=\frac{\varphi}{\tau}\left(f\left(v_{i, j}^{x}\right)-n_{i, j}^{x}+I_{0}+I_{\text {stim }}^{x}\right), \\
\frac{d n_{i, j}^{x}}{d t}=\frac{1}{\tau}\left(g\left(v_{i, j}^{x}\right)-n_{i, j}^{x}\right), \\
f\left(v_{i, j}^{x}\right)= \begin{cases}a_{n}\left(v_{i, j}^{x}+b_{n}\right)^{2}-c_{n} & \text { when } v_{i, j}^{x}<0, \\
-a_{p}\left(v_{i, j}^{x}-b_{p}\right)^{2}+c_{p} & \text { when } v_{i, j}^{x} \geq 0,\end{cases} \\
g\left(v_{i, j}^{x}\right)= \begin{cases}k_{n}\left(v_{i, j}^{x}-p_{n}\right)^{2}+q_{n} & \text { when } v_{i, j}^{x}<r, \\
k_{p}\left(v_{i, j}^{x}-p_{p}\right)^{2}+q_{p} & \text { when } v_{i, j}^{x} \geq r,\end{cases}
\end{gathered}
$$

where variables $v_{i, j}^{x}, n_{i, j}^{x}$ and $I_{s t i m}^{x}, x=1,2$ are the membrane potential, a slow variable, the stimulus input of the neurons in layer 1 and 2 and they are correspond to the pixel $(i, j)$ in image. Variable $I_{0}$ is the bias constant. Parameters $\varphi$ and $\tau$ are time constants. Parameters $r, a_{x}, b_{x}, c_{x}, k_{x}, p_{x}, q_{x}$ for $x=n$ and $\mathrm{p}$, are constants that control the silicon neuron's dynamics. Please see appendix for the parameter settings of the Class I and II modes.

Our silicon synapse model based on the kinetic model in [13] describes the transmitter release and the post-synaptic current generation [14]. Its equation is,

$$
\frac{d I_{s_{i, j}}^{x}}{d t}=\left\{\begin{array}{cc}
\alpha\left(1-I_{s_{i, j}}^{x}\right) & {[T]=1,} \\
-\beta I_{s_{i, j}}^{x} & {[T]=0,}
\end{array}\right.
$$

where, $I_{s_{i, j}}^{x}$ and $[T]$ denote the post-synaptic current and the amount of the released transmitter per pre-synaptic spike, respectively. We defined $[T]$ as $1(0)$ when the membrane potential of the pre-synaptic neuron is over (under) a threshold. Parameters $\alpha$ and $\beta$ are the forward and backward rate constants which describe the transition rates of the receptors between its closed state and open state.

The stimulus input of neurons (N1), $\mathrm{I}_{\mathrm{stim}}^{1}$, is calculated as follows:

$$
I_{s t i m_{i, j}}^{1}=c^{1} x_{i, j}
$$

where, $x_{i, j}$ is the value of pixel $(\mathrm{i}, \mathrm{j})$ in the gray scale image. $c^{1}$ is a constant to scale the stimulus input. The stimulus input of neurons $\left(\mathrm{N}^{2}\right), I_{s t i m_{i, j}}^{2}$, is governed by:

$$
I_{s_{t i m}, j}^{2}=c^{2} W_{x}\left(\begin{array}{ccc}
I_{s_{i-1, j-1}}^{1} & I_{s_{i-1, j}}^{1} & I_{s_{i-1, j+1}}^{1} \\
I_{s_{i, j-1}}^{1} & I_{s_{i, j}}^{1} & I_{s_{i, j+1}}^{1} \\
I_{s_{i+1, j-1}}^{1} & I_{s_{i+1, j}}^{1} & I_{s_{i+1, j+1}}^{1}
\end{array}\right)
$$

where, $c^{2}$ is a constant. $W_{x}(\mathrm{x}=\mathrm{on}$ and off $)$ are the synaptic matrices which represent the receptive fields of On-center and Off-center neuron, respectively.

\section{Hardware Implementation}

We build our silicon neuronal network on FPGA device. The neuron and synapse models shown in Eq.(1)-(5) are solved by Euler method. The step size is 0.000375 . The values of parameters are 
selected as power-of-2 numbers to reduce the hardware utilization. Therefore, the multiplications are replaced by shift operations.

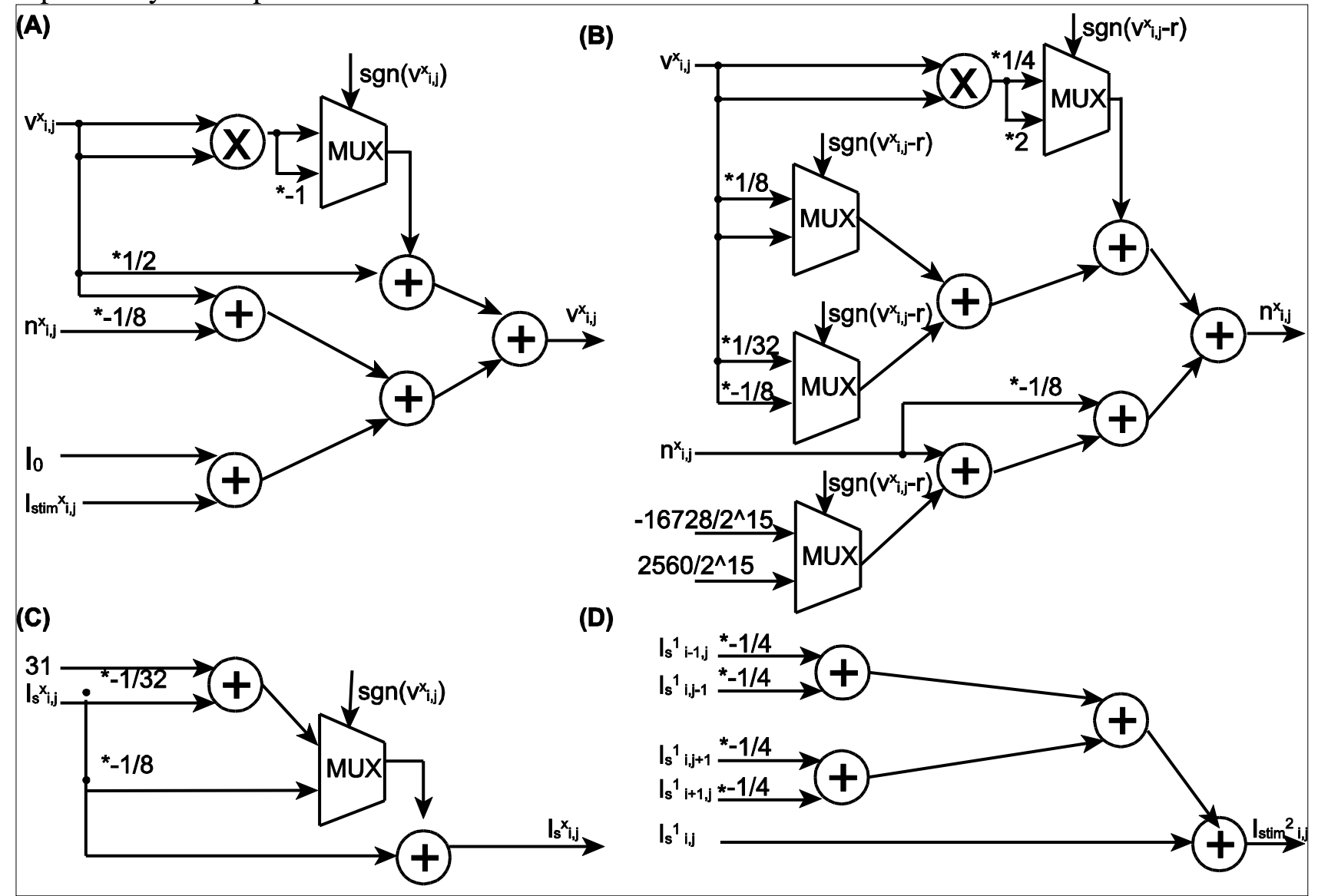

Fig. 4. Block diagrams of the $v_{i, j}^{\mathrm{x}}(\mathrm{A}), \mathrm{n}_{\mathrm{i}, \mathrm{j}}^{\mathrm{X}}(\mathrm{B}), \mathrm{I}_{\mathrm{s}, \mathrm{j}}^{\mathrm{x}}(\mathrm{C})$ and $\mathrm{I}_{\mathrm{stim}}^{2}(\mathrm{D})$ circuits.

Figure 4(A,B) show the block diagrams of $v_{i, j}^{x}$ and $n_{i, j}^{x}$ in DSSN model and the silicon neuron is in Class I. Symbols $\times,+$ and MUX in the figure represent a multiplier, an adder and a multiplexer, respectively. A multiplexer selects one of input signals by the control signal and forwards the selected input into the output port. Values marked with * represent the multiplication that is realized by a right or a left shift operation. A multiplier is shared by all the $\times$ because their inputs are same. The DSSN model costs 1 multiplier, 10 adders and 5 multiplexers. Figure $4(C)$ shows the block diagram of $I_{s_{i, j}}^{x}$ in silicon synapse model. It costs 2 adders and 1 multiplexer. The $\operatorname{stimulus} \operatorname{input}\left(I_{s t i m_{i, j}}^{1}\right)$ is fed to our network directly as the input, while $\operatorname{input}\left(I_{\text {stim }}^{2}\right)$ calculated by Eq.(7) is realized by 4 adders as shown in Fig.4(D) when we set $\mathrm{W}_{\text {on }}=\{0,-1,0 ;-1,4,-1 ; 0,-1,0\}$

\section{Simulation Results}

We simulated our silicon neuronal network with $\mathrm{C}++$ programs to estimate its performance of edge detection. The gray image shown in Fig.5(A) with $512 \times 512$ pixels is presented to our network. The neuron $\mathrm{N}^{1}$ responds to stimulus input governed by Eq.(6). Constant $c^{1}$ is 0.0625 . The synaptic matrices $\mathrm{W}_{\text {on }}$ and $\mathrm{W}_{\text {off }}$ simulating the On-center and Off-center type receptive fields are defined as $\{0,-1,0 ;-1,1,-1 ; 0,-1,0\}$ and $\{0,0.25,0 ; 0.25,-1,0.25 ; 0,0.25,0\}$, respectively. The stimulus input for neuron $\mathrm{N}^{2}$ is obtained by multiply the post-synaptic currents as shown in Eq.(7). The constant $c^{2}$ is 0.25 . We calculate the firing rate of $\mathrm{N}^{2}$ and normalize it to denote the edge information. The high rate denoted by bright spot refers to the edge with high contrast. The low rate denoted by the dark spot refers to the edge with low contrast. Figure $5(B, C)$ are the firing rate map of our silicon neuronal network when On-center and Off-center receptive fields are configured, respectively. As we can see, the results of both On-center type and Off-center type show the edge information of the original 
image. However, both of them detect some fake edges and lose some real edges. The On-center type detects more details than the Off-center. The results of Laplacian operation are shown in Fig.5(D).

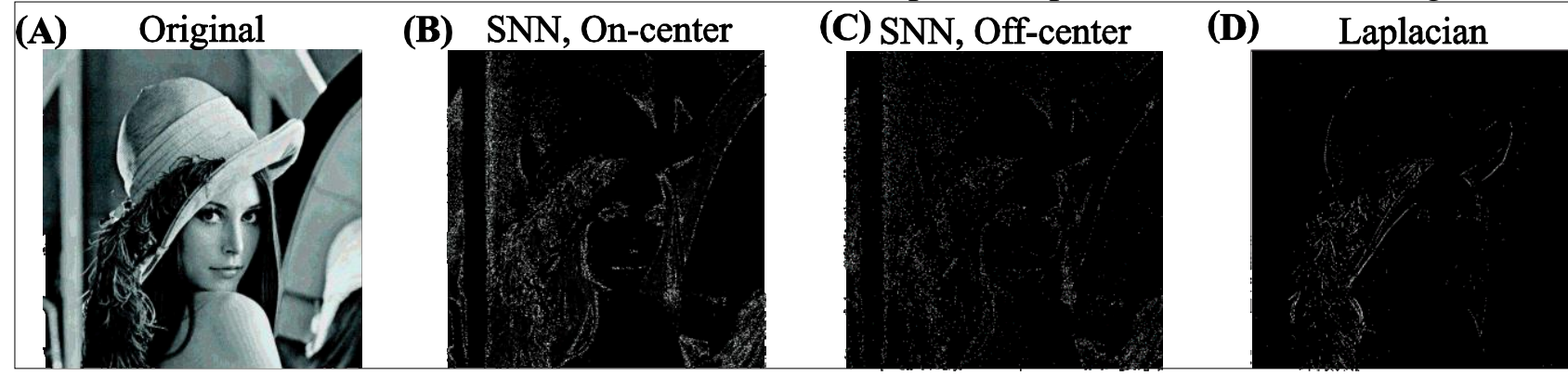

Fig. 5. Silicon neuronal network(SNN) for edge detection and Laplacian operation. Plane (A) is the original image. Planes $(B, C)$ are the firing rate map of silicon neuronal network with On-center and Off-center type receptive fields. Plane (D) is the result of Laplace operation.

\section{Discussion}

Our silicon neuronal network simulated the mechanism of signal processing in the retina. The ganglion neuron is described by the DSSN neuronal model which shows various neuronal behaviors. And the silicon synapse model is used to represent the precise behaviors of the neuron and to realize On-center and Off-center receptive fields. Our silicon neuronal network is optimally designed for the hardware implementation. Its parameters are selected to power of two numbers to save the cost. The edge detection is successful in our network when silicon neurons included in it are configured into Class I. However, our network also detects some fake edges and loses some real edges because of the influence of image noise.

In the future, we will improve the edge detection algorithm of our network. We will also finish the VHDL design and download our silicon neuronal network to an FPGA device and evaluate its functionality. The network including Class II silicon neurons will be considered. Biological experiments indicate that signals are fed to the cortex after the process of retina. The simple cells in cortex classify signals and serve as the function of orientation selection. We will use our silicon neuronal network performing complex recognition task based on the mechanism of simple cells. And consider its synaptic plasticity and learning ability as well.

\section{References}

[1] T.F. Smith and M,S. Waterman, Identification of common molecular subsequences, J. Mol. Biol. 147, 1981, pp. 195-197.

[2] K.A. Zaghloul and K. Boahen, A silicon retina that reproduces signals in the optic nerve, Journal of neural engineering 3(4), 2006, pp. 257-267.

[3] P. Lichtsteiner, C. Posch and T. Delbruck., A 128x128 120dB 15us-Latency temporal contrast vision sensor, IEEE J. Solid-State Circuits 43(2), 2008, pp. 566-576.

[4] C. Bartolozzi, et al., Embedded neuromorphic vision for humanoid robots, IEEE Computer Society Conference on Computer Vision and Pattern Recognition Workshops (CVPRW), 2011, pp. 129-135.

[5] F. Gomez-Rodriguez, et al., Neuromorphic real-rime objects tracking using address event representation and silicon retina, Lecture Notes in Computer Science 6691, 2011, pp.133-140.

[6] C. Posch, D. Matolin and R. Wohlgenannt, A QVGA $143 \mathrm{~dB}$ DR asynchronous address-event PWM dynamic image sensor with lossless pixel-level video compression, IEEE ISSCC Dig. Tech. 2010, pp. 400-401. 
[7] F. Eibensteiner, J. Kogler and J. Scharinger, A high-performance hardware architecture for a frameless stereo vision algorithm implemented on a FPGA platform., IEEE Conference on Computer Vision and Pattern Recognition Workshops, 2014, pp. 637-644.

[8] B. Kim, H. Okuno, T. Yagi and M. Lee, Implementation of visual attention system using articial retina chip and bottom-up saliency map model, Lecture Notes in Computer Science 7064, 2011, pp. 416-423.

[9] L. Lapicque, Recherches quantitatives sur l'excitation electrique des nerfstraitee comme une polarization, J. Physiol. 9, 1907, pp. 620-635.

[10] Z. Zhuo, Q.X. Wu and Z. Zhang, GPU implementation of spiking neural networks for edge detection, In: D.-S. Huang et al. (Eds.) ICIC 2013. CCIS, vol. 375, 2013, pp.147-152.

[11] T. Kohno and K. Aihara, Digital spiking silicon neuron: concept and behaviors in GJ-coupled network, In: Proceedings of International Synposium on Arti_cial Life and Robotics, OS3-OS6. 2007, Beppu.

[12] A.L. Hodgkin, The local electric changes associated with repetitive action in a non-medullated axon, J. Physiol., 107, 1948, pp. 165-181.

[13] A. Destexhe, A.F. Mainen and T.J. Sejnowski, Kinetic models of synaptic transmission, Methods in Neuronal Modeling, eds. C.KochandI. Segev (Cambridge, FL:MITPress), 1998, pp. 1-25.

[14] J. Li, Y. Katori and T. Kohno, An FPGA-based silicon neuronal network with selectable excitability silicon neurons, Front Neurosci. 6, 183, 2012.

\section{Appendix}

Table 1 Common parameters for Class I and II neuron

\begin{tabular}{|c|l|c|l|}
\hline Par. & Value & Par. & Value \\
\hline $\mathrm{a}_{\mathrm{n}}$ & 8.0 & $\mathrm{k}_{\mathrm{p}}$ & 16 \\
\hline $\mathrm{b}_{\mathrm{n}}$ & 0.25 & $\mathrm{p}_{\mathrm{p}}$ & $2^{-5}-2^{-2}$ \\
\hline $\mathrm{c}_{\mathrm{n}}$ & 0.5 & $\mathrm{q}_{\mathrm{p}}$ & -0.6875 \\
\hline $\mathrm{a}_{\mathrm{p}}$ & 8.0 & $\tau$ & 0.003 \\
\hline $\mathrm{b}_{\mathrm{p}}$ & 0.25 & $\alpha$ & 83.3 \\
\hline $\mathrm{c}_{\mathrm{p}}$ & 0.5 & $\beta$ & 333.3 \\
\hline
\end{tabular}

Table 2 Different parameters for Class I and II neuron

\begin{tabular}{|c|l|l|l|l|l|}
\hline \multicolumn{2}{|l|}{ Par. } & Value & \multicolumn{2}{l|}{ Par. } & Value \\
\hline \multirow{2}{*}{$\mathrm{k}_{\mathrm{n}}$} & I & 2.0 & \multirow{4}{*}{$\varphi$} & I & 1.0 \\
\cline { 2 - 3 } \cline { 5 - 6 } & II & 4.0 & & II & 0.5 \\
\hline \multirow{2}{*}{$\mathrm{p}_{\mathrm{n}}$} & I & $-2^{-2}-2^{-4}$ & $\mathrm{r}$ & $\mathrm{I}$ & -0.205357142 \\
\cline { 2 - 3 } \cline { 5 - 6 } & II & $-2^{-1}-2^{-4}$ & & II & -0.104166 \\
\hline \multirow{2}{*}{$\mathrm{q}_{\mathrm{n}}$} & I & -0.705795601 & $\mathrm{I}_{0}$ & I & -0.205 \\
\cline { 2 - 3 } \cline { 5 - 6 } & II & -1.317708517 & & II & -0.23 \\
\hline
\end{tabular}

